auf das ernsthafteste gefährdeten“ (S. 141). Die ,,interne Lösung“ könnte zwar Niemanns Ergebnis bestätigen, daß nicht die Guerilla ein unabhängiges Zimbabwe schuf, sondern der gewaltfreie Widerstand des Bischofs Muzorewa zum Erfolg führte. Eine solche Einschätzung ist jedoch zweifelhaft, solange die ideologischen Differenzen und die dahinter stehenden Interessen zwischen der Patriotischen Front einerseits und dem Afrikanischen Nationalrat (ANC) andererseits in der Diskussion nicht berücksichtigt werden.

Trotz aufgezeigter Mängel bleibt die vorliegende Studie aufgrund der Verarbeitung umfänglichen Quellenmaterials für den fachlich interessierten Leser eine empfehlenswerte Lektüre.

Volker Kasch

Astri Suhrke/Lela Garner Noble (eds.)

\title{
Ethnic Conflict in International Relations
}

New York: Praeger 1977, 248 S.

Die Edition versucht in acht Fallstudien über Nord-Irland (T. G. Carroll), Zypern (N. Black), die Kurden im Iraq (G. S. Harris), den Libanon (L. Meo), Eritrea (Anonymus), die Kazakhen/China (J. T. Dreyer) und die Muslims in den Philippinen und Thailand (A. Suhrke/L. G. Noble) den Zusammenhang zwischen internen ethnischen Konflikten und den internationalen Beziehungen zu klären. Dabei wird insbesondere die Frage in den Vordergrund gestellt, ob nationale ethnische Konflikte besondere Anziehungskraft für ausländische Interventionen ausüben bzw. besonders leicht zu internationalen Konflikten sich auszuweiten drohen. Obwohl die Fallstudien - bis auf die über Nord-Irland - sich mit islamischen Volksgruppen (z. T. als Minderheiten) beschäftigen, für die ein internationales Interesse - etwa durch und über die ,,Islamische Konferenz der Außenminister" als Ausdruck des allgemein auch politischen ,,Wir"-Bewußtseins der Mohammedaner-am ehesten unterstellt werden könnte, werden auch hier nationale ethnische Konflikte von auswärtigen (islamischen) Mächten nur gelegentlich und zur Durchsetzung eigener - von den Konfliktursachen unabhängiger - Ziele aufgegriffen und für diese instrumentalisiert. Allgemeine theoretische Aussagen über die Strukturierung internationaler Konflikte bzw. Interventionen durch ethnische Auseinandersetzungen, um deren hypothetische Formulierung und Beantwortung die Herausgeber sich in einem längeren Vor- wie auch Nachwort durchaus verdient bemühen, können so offenbar kaum gemacht werden. Trotz dieser Fehlanzeige handelt es sich jedoch um ein lesenswertes Buch. Man hätte sich nur gewünscht, daß der Arbeitsbegriff „Ethnizität" theoretisch und empirisch (in den Fallstudien) etwas genauer geklärt worden wäre und daß der Zusammenhang zwischen dem Konfliktfeld ,Ethnizität“ mit der ökonomischen, politischen und ideologischen Dimension nicht nur kurz angesprochen (S. 8), sondern auch in den Fallstudien etwas ausführlicher herausgearbeitet worden wäre. Diese beschränken sich so auf eine (meist sehr lesenswerte) Schilderung der historischen Entwicklung der ,,internen“ und ,,externen“ Dimension der untersuchten Konflikte.

Rolf Hanisch

Michael P. Todaro

Internal migration in developing countries

A review of theory, evidence, methodology and research priorities

Geneva: International Labour Office, 1976, VI, 106 S., sfr. 20.-

Die in diesem Uberblick erfaßten rund 250 Titel sind fast ausschließlich englischsprachig und überwiegend in den letzten zehn Jahren entstanden. Als zentrales Problem stellt der Autor 